\title{
New timing solutions for RRATs
}

\author{
Bingyi Cui ${ }^{1}$, Jason Boyles ${ }^{1,2}$, Maura McLaughlin ${ }^{1}$ \\ and Nipuni Palliyaguru ${ }^{1}$ \\ ${ }^{1}$ Dept. of Physics, West Virginia University, Morgantown, WV 26506, United States \\ email: bcui@mix. wvu .edu \\ ${ }^{2}$ Dept. of Physics and Astronomy, Western Kentucky University, Bowling Green, KY 42101, \\ United States
}

\begin{abstract}
The rotating radio transients are sporadic pulsars which are difficult to detect through periodicity searches. By using a single-pulse search method, we can discover these sources, measure their periods, and determine timing solutions. Here we introduce our results on six RRATs based on Parkes and Green Bank Telescope (GBT) observations, along with a comparison of the spin-down properties of RRATs and normal pulsars.
\end{abstract}

Keywords. Pulsars: individual, stars: kinematics, stars: rotation

\section{Introduction}

Rotating Radio Transients (RRATs) are pulsars from which we detect only sporadic radio bursts, making them difficult to detect in traditional periodicity searches (McLaughlin et al. 2006). They were first discovered through single-pulse search reprocessing of the Parkes Multibeam Pulsar Survey data. Currently 70 of these sporadic pulsars are known. For these, we can calculate times-of-arrival and determine timing solutions, by using single pulses instead of the commonly used folded profiles. Timing solutions are crucial to understand their relation to other pulsars and the nature of their emission.

Here we introduce our results for six RRATs, along with a comparison between RRATs and normal pulsars. Five of these RRATs were discovered in a re-analysis of the Parkes Multibeam Survey data and one was discovered through a GBT drift-scan survey.

\section{Single-pulse search}

Classical search algorithms based on Fourier techniques or folding do not detect RRATs. In stead we search for individual pulses with signal-to-noise ratio above some threshold (typically $5 \sigma$ ) in a number of trial-DM time series. Then, once a RRAT is discovered, the first step in our timing analysis is to identify which pulses are from the RRAT. We do this by searching for pulses which are brighter at the DM of the RRAT than at zero DM. Figure 1 shows an example of the single-pulse search output for a nearly one-hour observation of PSR J1048-5838. We see very bright bursts peaking at DM of $69 \mathrm{pc} / \mathrm{cm}^{3}$ which differ from the signals peaking at $\mathrm{DM}$ of $0 \mathrm{pc} / \mathrm{cm}^{3}$ that are due to radio frequency interference (RFI). The pulsar only turns 'on' for six minutes of this observation.

\section{Timing solutions}

To get a timing solution for a RRAT, we must first calculate the spin period. We do this by measuring differences between pulse arrival times and calculating the greatest common denominator. Once a period is known, we bin the data into single pulse periods and calculate times-of-arrival (TOAs) as the peak for each detected pulse. We have calculated timing solutions for six RRATs. PSR J1048-5838 has the longest span of observation 


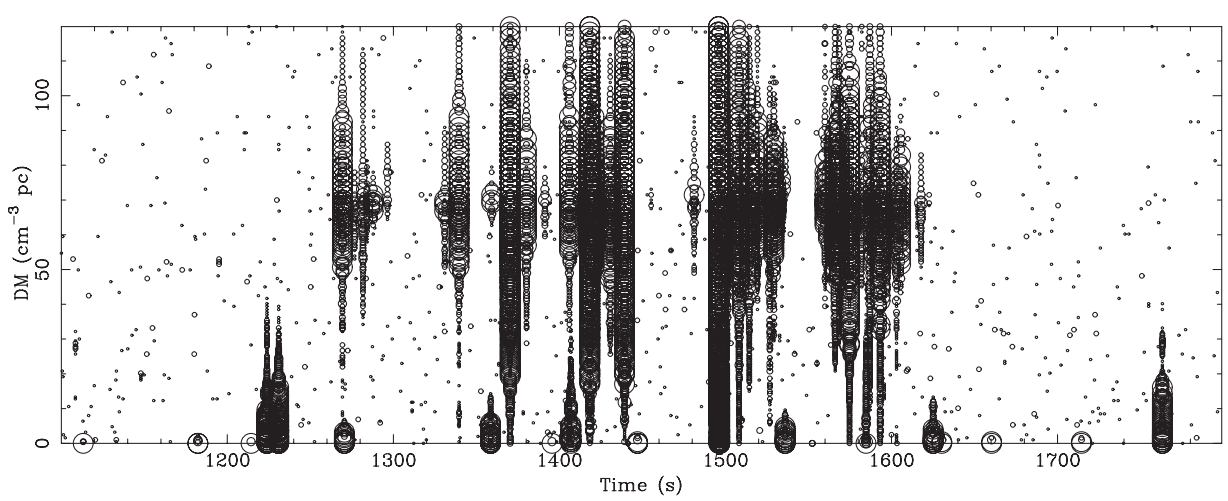

Figure 1. Single-pulse search plot for PSR J1048-5838. This plot shows a 16-minute portion of a 60 -minute Parkes $1.4 \mathrm{GHz}$ observation during which the RRAT is 'on'. Pulses are plotted at the detection DM and time, with size proportional to pulse signal-to-noise.

of these RRATs: four years of post-discovery timing observations and a 13-year span including the discovery. Note that this RRAT was found in an even later reprocessing of the Parkes Multibeam Survey after the initial RRATs (Keane 2010). The timing residuals shows good phase connection to its earlier discovery. We have calculated timing solution for this and five other RRATs. The periods are: 1.231s (PSR J1048-5838), 0.503 s (PSR J1623-0841), 1.818 s (PSR J1739-2521), 1.320 s (PSR J1754-3014), 0.933 s (PSR J1839-0141), and 0.414s (PSR J1848-1243). They have surface magnetic fields ranging from $4 \times 10^{11} \mathrm{G}$ to $4 \times 10^{12} \mathrm{G}$, spin-down luminosities ranging from $2 \times 10^{30} \mathrm{ergs}$ $\mathrm{s}^{-1}$ to $6 \times 10^{32} \mathrm{ergs} \mathrm{s}^{-1}$, and characteristic ages ranging from 1.6 Myr to $15 \mathrm{Myr} . \dagger$

\section{Pulse profiles and long timescale periodicities}

Profiles of the six RRATs are presented in Figure 2. Here, pulse profiles of PSRs J1739-2521 and J1839-0141 are sums of data during the $\sim$ minute-long time periods when the RRATs are 'on'. The profile of PSR J1048-5838 is a sum of all detected individual single pulses. The other profiles are created by folding each observation and summing all the profiles. Note that these three objects (PSRs J1623-0841, J1754-3014

$\dagger$ Latest RRAT solutions can be found in RRATalog: www.as.wvu.edu/ pulsar/rratalog

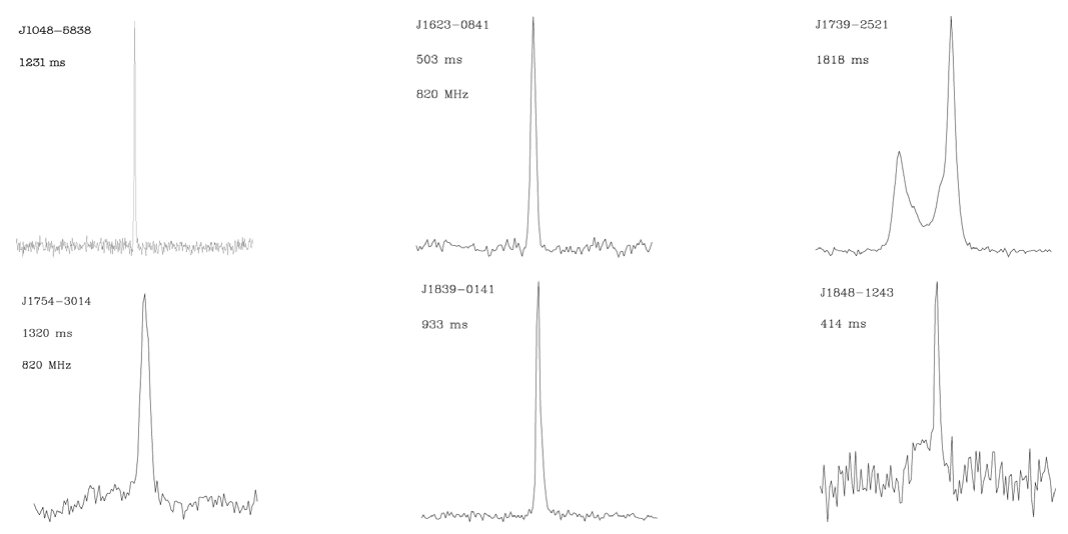

Figure 2. Pulse profiles of 6 RRATs. The profile of PSR J1048-5838 is based on $1.4 \mathrm{GHz}$ observations with Parkes telescope. The others are from $820-\mathrm{MHz}$ observations with the GBT. 


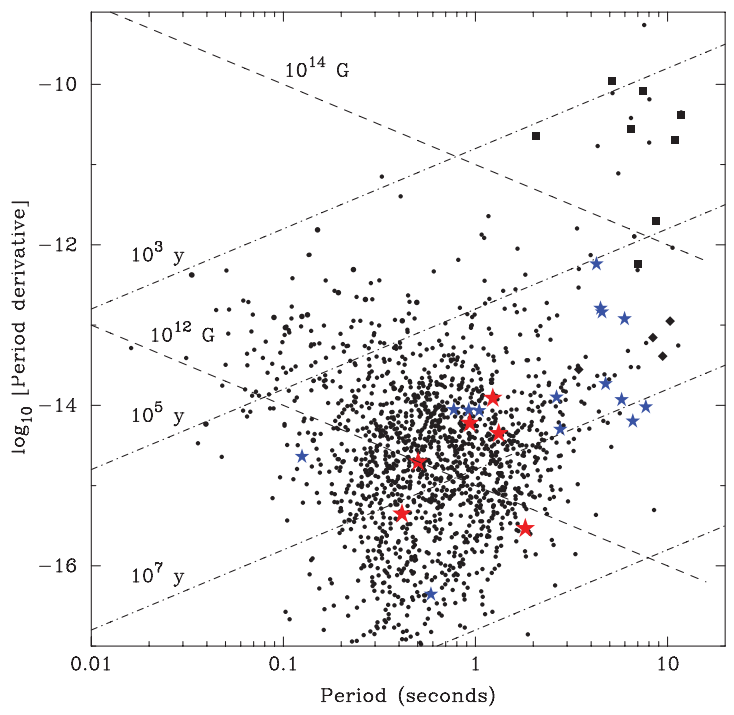

Figure 3. $P-\dot{P}$ diagram of solved RRATs and pulsars. New RRATs are shown as red stars and previously timed RRATs as blue stars. The black squares are magnetars, and black diamonds indicate x-ray isolated neutron stars. Lines of constant magnetic field (dashed) and characteristic age (dot-dashed) are shown. The KS test gives $1.12 \times 10^{-19}, 2.45 \times 10^{-4}, 1.94 \times 10^{-5}, 0.16$ and 0.04 probabilities that the period, period derivative, magnetic field, and characteristic age, spin-down energy-loss rate, respectively, were derived from the same distribution as those for other pulsars.

and J1848-1243) were detected through single-pulse searches but are typically detectable in follow-up observations by folding all of the data. It is clear that the RRATs are a diverse group of objects with varying properties.

We have applied Lomb-Scargle analysis (Scargle 1982) on the unevenly sampled pulse arrival times of PSR J1048-5838 to see if there are any periodicities in the timeseries. The spectrum gives the most significant periodic signal to be of period 19.15 hours with significance level of $1.95 \sigma$. This is much lower significance than the periodicities of similar timescale reported by Palliyaguru et al. (2011) for other RRATs.

\section{Population of RRATs: Why different?}

At this time, 21 of roughly 70 RRATs have timing solutions with period and period derivative, shown on the $P-\dot{P}$ diagram in Figure 3. We applied the Kolmogorov-Smirnov test (KS test) to the RRAT and normal pulsar populations to see how their spin-down properties compare (see Figure 3). The largest differences between the two groups are found in the distributions of period and magnetic field. While selection effects may be responsible for some of the period dependence, as longer period pulsars are more likely to be detected with higher signal-to-noise ratio in single-pulse searches (McLaughlin \& Cordes 2003), the difference in period derivative distributions hints that there is a fundamental difference in these populations.

\section{References}

Keane, E. F., Ludovici, D. A., Eatough, R. P., et al. 2010, MNRAS, 1057, 1068

Lorimer D. R., Kramer M. 2005, Handbook of Pulsar Astronomy (Cambridge University Press), McLaughlin, M. A. \& Cordes, J. M. 2003, ApJ, 982, 996

McLaughlin, M. A., Lyne, A. G., Lorimer, et al. 2006, Nature, 817, 820

Palliyaguru, N. T., McLaughlin, M. A., Keane, E. F., et al. 2011, MNRAS, 1871, 1880 